\title{
Asymptotic analysis of a doubly nonlinear diffusion equation
}

\author{
J. R. King \\ School of Mathematical Sciences \\ University of Nottingham \\ Nottingham NG7 2RD \\ England \\ john.king@nottingham.ac.uk
}

\begin{abstract}
We investigate the doubly nonlinear diffusion equation

$$
\frac{\partial u}{\partial t}=\frac{1}{n} \nabla \cdot\left(u^{m}|\nabla u|^{n-1} \nabla u\right)
$$

and the same equation expressed in terms of a 'pressure' variable. We classify various classes of compacted supported solutions, as well as finite-mass solutions that decay algebraically at infinity. A number of novel phenomena are identified, particularly for $n<0$, that seem to us worthy of further mathematical investigation.
\end{abstract}




\section{Introduction}

We are concerned in this paper with the doubly nonlinear equates

$$
\frac{\partial u}{\partial t}=\frac{1}{n} \nabla \cdot\left(u^{m}|\nabla u|^{n-1} \nabla u\right)
$$

which we shall also write without comment in the rescaled form

$$
\frac{\partial u}{\partial t}=\operatorname{sgn}(n) \nabla \cdot\left(u^{m}|\nabla u|^{n-1} \nabla u\right)
$$

depending on context. As the notation already implies, we consider only solutions with $u \geq 0$ everywhere. We shall be mainly concerned with finite-mass solutions and shall seek to explore the full parameter space of the exponents $(m, n)$. We have a number of distinct motivations. Firstly, (1.1) has two particularly well-studied special cases, namely the porous medium equation $(\mathrm{PME})^{1}$

$$
(n=1) \quad \frac{\partial u}{\partial t}=\nabla \cdot\left(u^{m} \nabla u\right)
$$

and the p-Laplace equation (PLE)

$$
(m=0) \quad \frac{\partial u}{\partial t}=\nabla \cdot\left(|\nabla u|^{n-1} \nabla u\right)
$$

see Vázquez [19], [20] for accounts of the extensive mathematical theory of these evolution equations. In one dimension there is an obvious relationship between (1.3) and (1.4), with $n=m+1$, but - as we shall see - the two can have very different qualitative properties; (1.1) allows the transition between the two be explored. Secondly, (1.1) has a number of physical applications, though our focus will not be on these. Finally, and perhaps most significantly here, equations of a similar type to (1.1) have been adopted in image-analysis applications, motivating a detailed exploration of the range of qualitative properties that (1.1) can exhibit and we emphasis in advance that there are regimes of $(m, n)$ parameter space in which phenomena not shared by (1.3) or (1.4) arise.

When $n<0$ we shall restrict ourselves to the one-dimensional case for the following reason, in effect well known in the image-analysis context: adopting the local expansion

$$
u \sim u_{0}+u_{1} x_{1}+U
$$

(1.1) implies in $N$ dimensions and at leading order that

$$
\frac{\partial U}{\partial t}=u_{0}^{m}\left|u_{1}\right|^{n-1}\left(\frac{\partial^{2} U}{\partial x_{1}^{2}}+\frac{1}{n}\left(\frac{\partial^{2} U}{\partial x_{2}^{2}}+\ldots+\frac{\partial^{2} U}{\partial x_{N}^{2}}\right)\right),
$$

i.e. $U$ satisfies for $n<0$ a forward heat equation in the direction of the gradient of $u$ but a backward one in directions orthogonal to that gradient. In addition, in one dimension only can

\footnotetext{
${ }^{1}$ We retain the PME and PLE terminology whatever the values (and in particular the signs) of the exponents $m$ and $n$ in (1.3) and (1.4).
} 
more general formulations such as

$$
\frac{\partial w}{\partial t}=\frac{1}{\mu} w^{\alpha}\left|\frac{\partial w}{\partial x}\right|^{\beta} \frac{\partial}{\partial x}\left(w^{\gamma}\left|\frac{\partial w}{\partial x}\right|^{\mu-1} \frac{\partial w}{\partial x}\right)
$$

be reexpressed in the form (1.1): setting

$$
w=\phi^{\mu /(\gamma+\mu)}
$$

enables (1.5) to be written, up to rescaling, in the form

$$
\frac{\partial \phi}{\partial t}=\frac{1}{n} \phi^{\sigma} \frac{\partial}{\partial x}\left(\left|\frac{\partial \phi}{\partial x}\right|^{n-1} \frac{\partial \phi}{\partial x}\right)
$$

where $n=\mu+\beta, \sigma=(\gamma(1-\beta)+\alpha \mu) /(\gamma+\mu)$, and (1) follows on introducing $u=\phi^{1-\sigma}$.

The remainder of the paper is organised as follows. In Section 2 we outline a number of preliminary results that serve to guide the detailed analyses in Section 3 of $n>0$ and in Section 4 of $n<0$. Sections 3 and 4 each include a summary of the main results therein, so we conclude in Section 5 with a brief discussion. Appendix 1 notes some equivalence transformations that are of independent interest and can be exploited in mapping some of the IBVPs that we explore into ones studied in other contexts; we do not pursue this avenue here, however, preferring to focus on (1.1) as it stands, except in Section 4.4. Appendix 2 addresses the exceptional (borderline) case $n=0$. Our analysis will be formal throughout and will sidestep some basic mathematical questions about the properties of the governing equations. We should remark at the outset that our discussion in what follows is in places rather speculative, and innumerable issues remain open (notably, but far from only, in higher dimensions), even at the formal level. We have attempted to keep the analysis fairly self-contained, and apologise for where we have failed to reference (or have inadequately referred to) existing literature.

\section{Classification of singularities}

\subsection{Preamble and Barenblatt solutions}

This section is concerned with the one-dimensional problem in the form

$$
\frac{\partial u}{\partial t}=\frac{1}{n} \frac{\partial}{\partial x}\left(u^{m}\left|\frac{\partial u}{\partial x}\right|^{n-1} \frac{\partial u}{\partial x}\right),
$$

having degeneracies both at $u=0$ and at $\partial u / \partial x=0$ (as well as where each becomes unbounded), which we need to characterise in order to classify possible solutions. Since we are interested in finite-mass solutions, a natural starting point is the Barenblatt solutions [1] that both examplify many of the possible singularities and are candidates for the large-time asymptotic behaviour for most regimes of the exponents $m$ and $n$. We refer to [9], for example, for further background on, and results for, (2.1). 
The self-similar form that conserves mass,

$$
\int_{-\infty}^{\infty} u(x, t) d x=M
$$

for constant $M$, takes the form

$$
u(x, t)=t^{-\alpha} f(\eta), \eta=x / t^{\alpha}, \alpha=1 /(m+2 n) .
$$

In the first instance considering even solutions, $f(\eta)=f(-\eta)$, we limit attention to $\eta>0$ with $d f / d \eta<0$ so that, on integrating once,

$$
f^{\frac{m-1}{n}} \frac{d f}{d \eta}=-\left(\frac{n}{m+2 n}\right)^{\frac{1}{n}} \eta^{\frac{1}{n}} \text { in } \eta>0
$$

requiring in particular that $n$ take the same sign as $m+2 n$ (for the 'finite-time' ansatz

$$
u(x, t)=\left(t_{c}-t\right)^{-\alpha} f\left(x /\left(t_{c}-t\right)^{\alpha}\right)
$$

they must take opposite signs; this version of self-similarity can, however, be shown to generate no viable finite-mass solutions). Proceeding from (2.4), we can identify the following regimes. In what follows we repeat for clarity equivalent functional forms in slightly different guises; in each case $a$ is a positive constant that can be determined from the initial data via (2.2). We omit borderline cases.

(I) $n>0, m>1-n(m>-2 n$ is then ensured).

$$
f^{(m+n-1) / n}=\left(\frac{n}{m+2 n}\right)^{\frac{1}{n}} \frac{m+n-1}{n+1}\left(a^{\frac{n+1}{n}}-\eta^{\frac{n+1}{n}}\right)_{+} .
$$

This regime is the most conventional one, exhibiting bounded solutions with compact support; it includes the usual slow-diffusion PME $(n=1, m>0)$ and PLE $(n>1, m=0)$ cases.

(II) $n>0,-2 n<m<1-n$.

$$
f^{-(1-m-n) / n}=\left(\frac{n}{m+2 n}\right)^{\frac{1}{n}} \frac{1-m-n}{n+1}\left(a^{\frac{n+1}{n}}+\eta^{\frac{n+1}{n}}\right) .
$$

In this regime, $f$ is bounded and decays algebraically (with finite mass) as $\eta \rightarrow+\infty$. It encompasses the usual fast diffusion ranges of PME $(n=1,-2<m<0)$ and PLE $(0<n<$ $1, m=0)$.

$$
\begin{aligned}
& \text { (III) }-1<n<0, m<-2 n(m<1-n \text { is then ensured }) . \\
& f^{(m+n-1) / n}=\left(\frac{n}{m+2 n}\right)^{\frac{1}{n}} \frac{1-m-n}{n+1}\left(\eta^{\frac{n+1}{n}}-a^{\frac{n+1}{n}}\right)_{+} .
\end{aligned}
$$

Here, and in the two cases that follow, $\alpha$ is negative in (2.3) so that solutions approach a delta function as $t \rightarrow \infty$ rather than spreading out. (2.7) has compact support but is unbounded (but of finite mass) at the origin. 
(IV) $n<-1, m<1-n(m<-2 n$ is then ensured).

(2.5) is then again an appropriate expression and the characterisation is as in (I), though we stress that $\alpha<0$ in (IV) implies that the solution is contracting, in contrast to expansion $(\alpha>0)$ in $(\mathrm{I})$.

(V) $1-n<m<-2 n$ ( $n<-1$ is then ensured).

Similarly, (2.6) is again applicable in this case.

The finite-mass condition on (II) is that $m+2 n>0$, while in (III) and (V) it is $m+2 n<0$ : these finite-mass requirements are accordingly automatically satisfied given the constraints on $(m, n)$ imposed by the form of the explicit solution.

Cases (I)-(V) appear to classify the full set of regimes over which the similarity reduction (2.3) is appropriate in the sense of generating plausible (large-time) finite-mass solutions. It remains next to characterise them more fully in the context of fully time-dependent solutions and to investigate the cases not captured by them, namely (VI) $n>0, m<-2 n$; (VII) $-1<n<0,-2 n<m<1-n$; (VIII) $-1<n<0, m>1-n$ ( $m>-2 n$ is then ensured); and (IX) $n<-1, m>-2 n(m>1-n$ is then ensured). The exceptional role of $n=-1, m=2$ at the intersection of many of these regimes should already be apparent.

In the above we have, for good reason, focussed on the one-dimensional case. The generalisation to higher dimensions with radial symmetry is, however, sufficiently straightforward for the results to be worth recording also at this point; in $N$ dimensions (2.3) becomes

$$
u(r, t)=t^{-N \propto} f(\eta), \eta=r / t^{\alpha}, \alpha=1 /((m+n-1) N+n+1)
$$

and the only other changes are as follows: wherever the combination $m+2 n$ of $m$ and $n$ appears in (2.4)-(2.7) it generalises to $(m+n-1) N+n+1$, while in (II) the lower bound on $m$, and in (III) and (V) the upper one, becomes $1-n-(1+n) / N$.

\subsection{Behaviour as $u \rightarrow 0^{+}$}

\subsubsection{Finite speed of propagation}

In the case of compactly supported solutions with $u \equiv 0$ for $x>s(t)$, the dominant local balance arises from the convective term dominating the time derivative in the frame of reference of the moving interface i.e., imposing mass conservation when integrating,

$$
\dot{s} u \sim \frac{1}{n} u^{m}\left|\frac{\partial u}{\partial z}\right|^{n-1} \frac{\partial u}{\partial z} \quad \text { as } z \rightarrow 0^{+}
$$

where $x=s(t)-z$. Hence

$$
u^{\frac{m+n-1}{n}} \sim(n \dot{s})^{\frac{1}{n}} \frac{m+n-1}{n} z \quad \text { as } z \rightarrow 0^{+} .
$$

This expression makes sense in two distinct cases, namely

$$
\text { (I) } n>0, m>1-n \text {, implying } \dot{s}>0 \text {, }
$$


(IV) $n<0, m<1-n$, implying $\dot{s}<0$.

Such behaviour is potentially relevant in higher dimensions also, the leading-order local behaviour at an interface typically being given by a one-dimensional balance.

\subsubsection{Behaviour as $x \rightarrow+\infty$}

There are two natural candidates in this limit. Firstly, the separable balance

$$
u \sim A\left(t x^{-(n+1)}\right)^{1 /(1-m-n)} \text { as } x \rightarrow+\infty
$$

requires that the constant $A>0$ be given by

$$
A^{1-m-n}=\left(\frac{n+1}{1-m-n}\right)^{n} \frac{m+2 n}{n},
$$

consistent with (2.6). This makes sense (in particular being of finite mass) for

(II) $n>0,-2 n<m<1-n$,

(V) $n<-1,1-n<m<-2 n$;

(2.12) is then, respectively, increasing and decreasing with $t$.

A finite-mass separable form is also possible for (VII) $,-1<n<0,-2 n<m<1-n$, but this then needs to be of finite-time extinction type

$$
u \sim A\left(\left(t_{c}-t\right) x^{-(n+1)}\right)^{1 /(1-m-n)}, A^{1-m-n}=\left(\frac{n+1}{1-m-n}\right)^{n} \frac{m+2 n}{-n},
$$

being associated with initial data with the specific power-law decay implied by (2.14).

The second possibility involves a quasi-steady balance

$$
u^{m}\left|\frac{\partial u}{\partial x}\right|^{n-1} \frac{\partial u}{\partial x} \sim-n J \text { as } x \rightarrow+\infty
$$

where $J(t)$ is the flux of mass to infinity. Thus

$$
u^{\frac{m+n}{n}} \sim-(n J)^{\frac{1}{n}} \frac{m+n}{n} x \text { as } x \rightarrow+\infty ;
$$

finite mass and, equivalently, self-consistency in neglecting the $\partial u / \partial t$ term in deriving (2.15) requires one of the following to hold:

$$
\begin{aligned}
& \text { (A) }-2 n<m<-n \text { (requiring } n>0 \text { and } J>0 \text { ); } \\
& \text { (B) }-n<m<-2 n \text { (requiring } n<0 \text { and } J<0 \text { ). }
\end{aligned}
$$

The behaviour (2.16) leads to an underspecified problem since an arbitrary uniform term $K(t)$ can be included on the right-hand side of (2.16), i.e. there are two degrees of freedom as $x \rightarrow+\infty$ (namely $J(t)$ and $K(t)$ ): generating a correctly specified problem would require that $J(t)$, say, be specified. (A) is a subcase of (II) above and for $n=1$ corresponds to the non-maximal solutions of PME from [8].

The above asymptotic forms (local to an interface in the case of (2.8) and far-field for (2.12), (2.16), as well as for (2.14)) will play a central role in our analysis and we shall repeatedly revisit them. We shall also, however, also need to investigate a number of other solution branches, as well as in Section 3 noting some higher-dimensional generalisations. 


\section{$3 n>0$}

\subsection{Preliminaries}

Much of the behaviour here rather closely follows that of PME and we shall be fairly brief in presenting results. It is both expedient and instructive to work in terms of two equivalent formulations, namely the original one,

$$
\frac{\partial u}{\partial t}=\nabla \cdot\left(u^{m}|\nabla u|^{n-1} \nabla u\right),
$$

and a 'pressure' version whereby

$$
p=\left|\frac{n}{m+n-1}\right|^{\frac{n-1}{n}} u^{\frac{m+n-1}{n}}
$$

so that

$$
\frac{\partial p}{\partial t}=p \nabla \cdot\left(|\nabla p|^{n-1} \nabla p\right)+\frac{n}{m+n-1}|\nabla p|^{n+1} .
$$

We note that, whereas the parameters $m$ and $n$ each appear in (3.1) as exponents, in (3.2) $m$ appears only in a coefficient and it is at times helpful to work in terms of

$$
\gamma \equiv-\frac{n}{m+n-1}
$$

cf. [5], for example, for $n=1$; the Barenblatt solution takes a particularly simple form in terms of $p$ and (3.1) is equivalent to

$$
\frac{\partial u}{\partial t}+\nabla \cdot(u \boldsymbol{v})=0, \boldsymbol{v}=-\frac{n}{m+n-1}|\nabla p|^{n-1} \nabla p,
$$

i.e. the velocity $\boldsymbol{v}$ is related to the pressure $p$ by a nonlinear Darcy relationship. Moreover, for $\gamma>0$ (so that $p=0$ when $u$ is unbounded, and vice versa), equation (3.3) is of independent interest: see below.

A radially symmetric separable solution to (3.3) that genralises (2.12) is easily derived in the form

$$
p^{n}=n^{n-1}(1-m-n) r^{n+1} /(n+1)^{n}(m N-(1-n)(m N-(1-n) N+n+1) t,
$$

having the requirement

$$
1-n-(1+n) / N<m<1-n
$$

on signs, these inequalities also being implied by the constraint that $u$ have finite mass when its behaviour as $r \rightarrow \infty$ is described by (3.6).

A quasi-steady balance in (3.1), whereby

$$
r^{N-1} u^{m}\left|\frac{\partial u}{\partial r}\right|^{n-1} \frac{\partial u}{\partial r} \sim-J(t)
$$


yields for $J>0$ that

$$
u^{\frac{m+n}{n}} \sim \frac{m+n}{N-n-1} J^{\frac{1}{n}} r^{\frac{n+1-N}{n}}+K(t)
$$

as a generalisation of (2.16). For $N<n+1, K(t)$ is arbitary and, as a far-field expression, (3.8) contains two degrees of freedom, leading to the non-maximal solutions noted below; conversely, for $N>n+1$ self-consistency demands $K(t) \equiv 0$ and

$$
u \sim\left(\frac{m+n}{N-n-1}\right)^{\frac{n}{m+n}} J^{\frac{1}{m+n}} r^{-\frac{N-n-1}{m+n}} \text { as } r \rightarrow \infty
$$

furnishes the relevant far-field behaviour. An additional self-consistency requirement on (3.8) that the left-hand side of (3.1) can indeed be neglected as $r \rightarrow \infty$ leads to

$$
\frac{N-n-1}{m+n}>N
$$

which is also the condition that (3.9) be of finite mass, so the constraints on exponents read

$$
\left(\mathrm{A}^{*}\right) N<n+1, \quad 1-n-(1+n) / N<m<-n \text {; }
$$

$\left(\mathrm{B}^{*}\right) N>n+1, \quad-n<m<1-n-(1+n) / N$.

The highly exceptional case $(m, n)=(-(N-1), N-1)$ is signposted by these. They generalise $(\mathrm{A})$ and $(\mathrm{B})$ above, respectively, but we stress with regard to $\left(\mathrm{B}^{*}\right)$, which requires $K(t) \equiv 0$, that we are concerned in this section only with $n>0$.

\subsection{The Cauchy problem for (3.1)}

For $m>1-n$ the large-time behaviour of the Cauchy problem is given by compactly supported Barenblatt solution noted above and we say no more about this case. When (3.7) holds, a Barenblatt solution, having algebraically decaying far field, is again available, but when $N<n+1$ non-maximal solutions, losing mass to infinity via the far-field behaviour (3.9), are also present when (3.10) (which is a subcase of (3.7)) is satisfied: these generalise those of PME described in [8] (and need not be restricted to $N=1$ ); because $J(t)$ and $K(t)$ are then both arbitrary in (3.8), these solutions are underspecified, necessitating that some relationship between $J(t)$ and $K(t)$ be prescribed. Conversely, when (3.11) applies it (being disjoint from (3.7)) represents a regime of correctly specified finite-mass solutions (generalising those of PME in [14], for example) that lose mass to infinity and presumably extinguish in finite time via a similarity solution of the second kind (we shall return to this below). For

$$
N<n+1, m \leq 1-n-(1+n) / N ; N=n+1, m<-n ; N>n+1, m \leq-n
$$

we conjecture that instantaneous extinction of finite-mass solutions occurs (cf. [18]).

While we shall not seek here fully to explore borderline cases, some brief remarks are in order. Firstly, on the borderline $m=1-n$ between compactly supported and algebraically decaying solutions, the large-time behaviour is of the form

$$
u \sim t^{-\frac{N}{n+1}} f(\eta), \eta=r / t^{\frac{1}{n+1}}
$$


with

$$
f(\eta)=A \exp \left(-\frac{n}{(n+1)^{(n+1) / n}} \eta^{\frac{n+1}{n}}\right)
$$

a profile that is independent of $N$ and generalises the Gaussian solution to the heat equation. Secondly, in considering $m_{c} \equiv 1-n-(1+n) / N$, we start with the radially symmetric form of $(3.1)$,

$$
\frac{\partial u}{\partial t}=-\frac{1}{r^{N-1}} \frac{\partial}{\partial r}\left(r^{N-1} u^{m}\left(-\frac{\partial u}{\partial r}\right)^{n}\right)
$$

given that $\partial u / \partial r \leq 0$, and set

$$
u(r, t)=r^{-N} c(x, t), x=\ln r,
$$

to give

$$
\frac{\partial c}{\partial t}=-\frac{\partial}{\partial x}\left(c^{m_{c}}\left(N c-\frac{\partial c}{\partial x}\right)^{n}\right) .
$$

The far-field of (3.13) is convectively dominated (cf. [14] for $n=1$ ), i.e.

$$
\frac{\partial c}{\partial t} \sim-N^{n} \frac{\partial}{\partial x}\left(c^{m_{c}+n}\right) \quad \text { as } \quad x \rightarrow+\infty
$$

so that

$$
c \sim\left(\frac{x}{N^{n}\left(m_{c}+n\right) t}\right)^{\frac{1}{m_{c}+n-1}} \text { as } \quad x \rightarrow+\infty,
$$

finite mass of $u$ then requiring that $0<m_{c}+n<1$, again necessitating that $N>n+1$, as might be expected. Finally, the most exceptional parameter set has $m=-(N-1), n=N-1$, as already alluded to above: this generalises the well-known case $N=2, m=-1, n=1$, with non-uniqueness occurring as for (3.10) but now with the maximal solution having positive (rather than zero) mass flux to infinity. Indeed, the calculation corresponding to that which leads to (3.9) gives

$$
u \sim \Phi(t) r^{-J^{\frac{1}{N-1}}(t)} \quad \text { as } \quad r \rightarrow \infty
$$

as a consistent far-field behaviour for arbitrary $\Phi(t) \geq 0$ and $0<J(t)<N^{N-1}$. The maximal solution has the minimal mass flux to infinity,

$$
J(t) \equiv N^{N-1},
$$

so that its extinction time can readily be calculated from the mass of the initial data and in this case (3.13) has the far-field limit

$$
\frac{\partial c}{\partial t} \sim N^{N-2}(N-1) \frac{\partial}{\partial x}\left(\frac{1}{c} \frac{\partial c}{\partial x}\right) \quad \text { as } \quad x \rightarrow+\infty,
$$

reproducing the familiar case with $N=2$. From (3.15) the maximal solution (which is uniquely specified) has

$$
c \sim 2 N^{N-2}(N-1) t / x^{2} \quad \text { as } \quad x \rightarrow+\infty .
$$




\subsection{Compactly supported solutions to (3.3)}

Here we investigate solutions to (3.3) akin to those for $n=1$ analysed in [5] (see also Section 9 of [7]). The results additionally provide insight into certain aspects of the case $n<0$.

For $m+n>1$ the transformation maps zeros of $u$ into those of $p$ and the local behaviour of the smoothest solutions is again given by the travelling-wave balance (3.17), and for these the large-time behaviour is as noted in the previous subsection. However, (3.3) is not in divergence form and it is natural also to consider solutions of the (one-dimensional) local form

$$
p \sim A(s, t) z^{\frac{m+n-1}{m+n}} \text { as } z \rightarrow 0^{+}
$$

where $z$ is in the inward normal distance from the interface, $p=0$, the latter being parameterised by $N-1$ coordinates $\boldsymbol{s}$. In (3.16), which arises from a quasi-steady limit in (3.3) and corresponds to Stefan-type solutions to (3.1) (cf. Section 3.4), $A \geq 0$ is arbitrary and the interface is a a priori unknown, leaving the problem underspecified. (3.16) amounts to the same balance as in (2.16), but no longer in the far-field: its self-consistency requirement for $n>0$ is that $m>-n$ but, since we require $p$ to be zero, at the interface we in fact require $m>1-n$. We note that (3.1) is less smooth than the conventional branch whereby (cf. (2.9))

$$
p \sim\left((m+n-1) V_{n} / n\right)^{1 / n} z \text { as } z \rightarrow 0^{+},
$$

wherein $V_{n}(>0)$ denotes the outward normal velocity of the interface. By contrast, (3.16) imposes no requirement on the sign of $V_{n}$.

For $m<1-n$ the situation is very different: (3.17) (which now requires $V_{n}<0$ ) is no longer the smoothest solution branch and is underspecified, a correction term

$$
B(s, t) z^{\frac{1-m}{1-m-n}}
$$

to (3.17) now being admissible for arbitrary $B$. For $m<1-n$ the smoothest solution branch has fixed front, $V_{n} \equiv 0$, and for $-2 n<m<1-n$ the local behaviour follows from (3.6) with $N=1$ i.e.

$$
p^{n} \sim n^{n-1}(1-m-n) z^{n+1} /(n+1)^{n}(m+2 n) t \text { as } z \rightarrow 0^{+} .
$$

The need for a transition at $m=-2 n$ is apparent from (3.18); for $n>0, m<-2 n$ the quasisteady balance (3.16) is admissible at a fixed front, though not at moving one, it now being correctly specified because the interface location is known from the initial data; it characterises the smoothest solutions. The powers of $z$ in (3.16) and (3.18) are equal at the transition value $m=-2 n$; there we have local behaviour

$$
p^{n} \sim n^{n-2} z^{n+1} \ln (1 / z) /(n+1)^{n-1} t \text { as } z \rightarrow 0^{+} .
$$

It is noteworthy that the above solution branches occupy the whole of $(m, n)$ parameter space in $n>0$ (there being everywhere one correctly specified solution branch and one singly 
underspecified one), with the sole exception of the homogeneous-in- $u$ borderline case $(m+n=$ 1), on which the behaviour differs significantly: here (3.2) should be replaced by

$$
p= \pm \ln u
$$

(corresponding to a Liouville-Green (JWKB) approach) to give

$$
\frac{\partial p}{\partial t}=\nabla \cdot\left(|\nabla p|^{n-1} \nabla p\right) \pm|\nabla p|^{n+1} .
$$

The smoothest solution branches for (3.21) is that in which the final term is negligible as $p \rightarrow 0^{+}$ (corresponding to $u \rightarrow 1$ ): this implies compactly supported solutions for $n>1$ but, strikingly (this being the only such case in the current subsection), not for $n \leq 1$. Underspecified branches exist with

$$
p \sim A(s, t) z \text { as } z \rightarrow 0^{+}
$$

with $A \geq 0$ arbitrary (in contrast to (3.17), $A$ cannot be expressed in terms of $V_{n}$ because the second term in (3.21) can enter locally via a contribution proportional to $z^{2}$ ).

On the smoothest solution branches, the large time behaviour is expected to be given by the Barenblatt solution for $m+n>1$, by the Barenblatt solution that neglects the final term of (3.21) when $m+n=1$ and by a separable solution

$$
p \sim t^{-\frac{1}{n}} g(\boldsymbol{x})
$$

when $m+n<1$.

\subsection{Stefan-type solution branches to (3.1)}

Here we consider moving-boundary problems on which the solution (3.1) loses mass at the interface, for example through a Stefan condition which would serve to relate $J$ in (3.23) below to $V_{n}$. The interface behaviour is again locally one-dimensional and follows from

$$
u^{m}\left(\frac{\partial u}{\partial z}\right)^{n} \sim J(s, t)
$$

for mass flux $J$ into the interface; thus

$$
u^{\frac{m+n}{m}} \sim \frac{m+n}{n} J^{\frac{1}{n}} z \text { as } z \rightarrow 0^{+}
$$

cf. (3.16). Such solutions are admissible for $m>-n$, i.e. for a wider range of exponents than that pertaining to mass-preserving compactly supported solutions (namely $m>1-n$; for $m>1-n$ they are of course equivalent to ones from Section 3.3). Under the conditions (3.10) this solution class can be continued into one that decays algebraically at infinity, as in (3.8); these correspond in some sense to having a Stefan condition imposed as $r \rightarrow \infty$. The borderline case $m=-n$ belongs to this latter class, though algebraic decay is replaced by exponential:

$$
u \sim \Phi(t) \exp \left(-n J^{\frac{1}{n}} r^{\frac{n+1-N}{n}} /(n+1-N)\right) \text { as } r \rightarrow \infty .
$$




\subsection{The Cauchy-Dirichlet problem}

Finally, we consider (3.1) on a bounded domain $\Omega$ with (cf. [17])

$$
u=0 \text { for } \boldsymbol{x} \in \partial \Omega \text {. }
$$

The local behaviour near $\partial \Omega$ is given by (3.16) requiring, in view of (3.2), that $m>-n$ (for $m \leq-n$ we conjecture that instantaneous extinction occurs). The simplest conjectures for the intermediate-asymptotic behaviour are then that

$$
\begin{aligned}
& u \sim t^{-\frac{1}{m+n-1}} f(\boldsymbol{x}) \text { as } t \rightarrow+\infty \text { for } m>1-n ; \\
& u \sim e^{-\lambda^{2} t} f(\boldsymbol{x}) \text { as } t \rightarrow+\infty \text { for } m=1-n ; \\
& u \sim\left(t_{c}-t\right)^{\frac{1}{1-m-n}} f(\boldsymbol{x}) \text { as } t \rightarrow t_{c}^{-} \text {for }-n<m<1-n .
\end{aligned}
$$

In the light of the corresponding PME results, (3.26) seems likely indeed to be applicable for all $m>1-n$, while for (3.27) we have

$$
\begin{array}{r}
\nabla \cdot\left(f^{1-n}|\nabla f|^{n-1} \nabla f\right)+\lambda^{2} f=0, f>0 \text { for } \boldsymbol{x} \in \Omega, \\
f=0 \text { for } \boldsymbol{x} \in \partial \Omega
\end{array}
$$

since (3.29) is invariant under rescalings of $f,(3.29)$ should be regarded as an eigenvalue problem from $\lambda$ (as is familiar when $n=1$ ).

The finite-time extinction case (3.28) is more subtle, however, as it is for PME (see [10], for example). Setting

$$
f=\alpha \phi^{\frac{n}{m+n}}, \alpha^{1-m-n} \equiv\left(\frac{n}{m+n}\right)^{n}(1-m-n)
$$

(so that $\phi \propto z$ as $z \rightarrow 0^{+}$) gives

$$
\begin{array}{r}
\nabla \cdot\left(|\nabla \phi|^{n-1} \nabla \phi\right)+\phi^{\mu}=0 \text { for } \boldsymbol{x} \in \Omega, \\
\phi=0 \text { for } \boldsymbol{x} \in \partial \Omega
\end{array}
$$

with $\mu \equiv n /(m+n)>n$. The PDE in (3.30) is the Euler-Lagrange equation for

$$
L=\int\left(\frac{1}{n+1}|\nabla \phi|^{n+1}-\frac{1}{\mu+1} \phi^{\mu+1}\right) d \boldsymbol{x}
$$

and the scaling invariant of (3.30) is a variational symmetry of (3.31) when

$$
\mu=\mu_{s} \equiv(N n+n+1) /(N-n-1),
$$

i.e.

$$
m=m_{s} \equiv-n((n-1) N+2(n+1)) /(n N+n+1),
$$


respectively generalising the well-known critical-Sobolev exponents

$$
\mu_{s}=(N+2) /(N-2), m_{s}=-4 /(N+2)
$$

for $n=1$. This variational symmetry allows the application of Noether's first theorem, to which we return shortly, and this in turn motivates the derivation of the following identities. Routine calculations on (3.30) yield

$$
\begin{aligned}
\nabla \cdot\left(\phi|\nabla \phi|^{n-1} \nabla \phi\right)-|\nabla \phi|^{n+1}+\phi^{\mu+1}=0 \\
\begin{aligned}
\left.\nabla \cdot((\boldsymbol{x} . \nabla \phi) \mid \nabla \phi)\right|^{n-1} \nabla \phi & \left.+\frac{1}{\mu+1} \boldsymbol{x} \phi^{\mu+1}-\frac{1}{n+1} \boldsymbol{x}|\nabla \phi|^{n+1}\right) \\
& +\left(\frac{N}{n+1}-1\right)|\nabla \phi|^{n+1}-\frac{N}{\mu+1} \phi^{\mu+1}=0
\end{aligned}
\end{aligned}
$$

so that if a solution to (3.30) exists then

$$
\begin{aligned}
& \int_{\Omega}|\nabla \phi|^{n+1} d \boldsymbol{x}=\int_{\Omega} \phi^{\mu+1} d \boldsymbol{x}, \\
& \left(\frac{N}{n+1}-1\right) \int_{\Omega}|\nabla \phi|^{n+1} d \boldsymbol{x}+\frac{n}{n+1} \int_{\partial \Omega} \boldsymbol{x} \cdot \hat{\boldsymbol{n}}\left(\frac{\partial \phi}{\partial z}\right)^{n+1} d S \\
& =\frac{N}{\mu+1} \int_{\Omega} \phi^{\mu+1} d \boldsymbol{x},
\end{aligned}
$$

where $\hat{\boldsymbol{n}}$ is the unit outward normal to $\partial \Omega$. Combining (3.36) and (3.37) (leading thereby to a generalisation of then usual Pohozaev identity) yields

$$
\frac{n}{n+1} \int_{\partial \Omega} \boldsymbol{x} \cdot \hat{\boldsymbol{n}}\left(\frac{\partial \phi}{\partial z}\right)^{n+1} d S=\left(\frac{N}{\mu+1}-\frac{N}{n+1}-1\right) \int_{\Omega} \phi^{\mu+1} d \boldsymbol{x} .
$$

The integral on the right-hand side is positive and it can be ensured that the left-hand side is also if $\Omega$ is star-shaped (by choosing the origin of $\boldsymbol{x}$ suitably). The identify (3.38) cannot then be satisfied if the coefficient of the integral is negative or zero i.e. if

$$
\mu \geq \mu_{s}, m \leq m_{s}
$$

For this criterion to come into play we require from (3.28) that $m_{s}>-n$, so that $N>n+1$ (we note that $m_{s}<1-n$ holds automatically). Hence for

$$
N<n+1 \text { with }-n<m<1-n ; N>n+1 \text { with } m_{s}<m<1-n
$$

we anticipate that (3.28) will indeed apply whereas for

$$
N>n+1 \text { with }-n<m<m_{s}
$$

we postulate that the second-kind solution to the Cauchy problem will be in the form of a shrinking spike

$$
u \sim\left(t_{c}-t\right)^{\alpha} f\left(\left|\boldsymbol{x}-\boldsymbol{x}_{c}\right| /\left(t_{c}-t\right)^{\beta}\right) \text { as } t \rightarrow t_{c}^{-},(1-n-m) \alpha+(n+1) \beta=1,
$$


for some $t_{c}, \boldsymbol{x}_{c}$ and with $\beta>0$ for $m<m_{s}$ and that this will also provide the intermediateasymptotic behaviour in the bounded domain case (cf. [10], [15], since the shrinking spike will be oblivious to the presence of the fixed boundary; for $m>m_{s}$ it will follow that $\beta<0$ and (3.39) will be inapplicable to bounded domains. For $N=n+1$, we have $m_{s}=-n$ and the exceptional status of $(m, n)=(-(N-1), N-1)$ for the Cauchy problem has already been noted.

Finally in this context, we need to address the borderline case $m=m_{s}$ : here the relevant separable solution can be obtained explicitly and we give two derivations below; this explicit form confirms the solution's role as the required second-kind solution to the Cauchy problem (having $\beta=0$ in (3.39) and hence consistent with $\beta>0$ for $m_{s}<m<1-n-(1+n) / N$ (note that $m_{s}<1-n-(1+n) / N$ holds for $\left.N>n+1\right)$ and $\beta<0$ for $-n<m<m_{s}$ ); suitable modulated by logarithmic terms (cf. [10]) it is also expected to describe the extinction behaviour in bounded domains. The first derivation we give is ad hoc but quick; the second is systematic, based on Noether's theorem. If setting

$$
\phi=\psi^{-\sigma}, \sigma=\frac{N-n-1}{n+1}
$$

and adopting radial symmetry with $\partial \phi / \partial r<0$ transforms (3.30) with $\mu=\mu_{s}$ to

$$
\sigma^{n}\left(-\frac{\psi}{r^{N-1}} \frac{\partial}{\partial r}\left(r^{N-1}\left(\frac{\partial \psi}{\partial r}\right)^{n}\right)+\frac{N n}{n+1}\left(\frac{\partial \psi}{\partial r}\right)^{n+1}\right)+1=0,
$$

a form in which the nonlinearities are most innocuous and, trying natural ansätze, leads to the explicit solution

$$
\psi=a\left(\frac{1}{N \sigma^{n}}+\frac{n}{n+1}\left(\frac{r}{a}\right)^{\frac{n+1}{n}}\right)
$$

wherein $a$ is an arbitrary constant that reflects the scaling invariance of the PDE (and the role of the second-kind solution as a nonlinear eigenfunction). Alternatively, combining (3.34) and (3.35) for $\mu=\mu_{s}$ yields (as dictated by Noether's theorem) the divergence form

$$
\begin{array}{r}
\nabla \cdot\left(\sigma \phi|\nabla \phi|^{n-1} \nabla \phi+(\boldsymbol{x} . \nabla \phi)|\nabla \phi|^{n-1} \nabla \phi-\frac{1}{n+1} \boldsymbol{x}|\nabla \phi|^{n+1}+\frac{\sigma}{N} \boldsymbol{x} \phi^{N / \sigma}\right) \\
=0,
\end{array}
$$

so, with radial symmetry,

$$
-\sigma \phi\left(-\frac{\partial \phi}{\partial r}\right)^{n}+\frac{n}{n+1} r\left(-\frac{\partial \phi}{\partial r}\right)^{n+1}+\frac{\sigma}{N} r \phi^{N / \sigma}=0
$$

a first integral that becomes

$$
\sigma^{n+1}\left(-\psi\left(\frac{\partial \psi}{\partial r}\right)^{n}+\frac{n}{n+1} r\left(\frac{\partial \psi}{\partial r}\right)^{n+1}\right)+\frac{\sigma}{N} r=0
$$

when rewritten in terms of $\psi$. Taking Legendre transforms yields

$$
\sigma^{n+1}\left(-\frac{1}{n+1} \hat{r}^{n+1} \frac{\partial \hat{\psi}}{\partial \hat{r}}+r^{n} \hat{\psi}\right)+\frac{\sigma}{N} \frac{\partial \hat{\psi}}{\partial \hat{r}}=0,
$$


so that

$$
\hat{\psi}=a\left(\frac{1}{n+1} \hat{r}^{n+1}-\frac{1}{N \sigma^{n}}\right),
$$

from which we immediately recover (3.41) on inversion.

It follows from (3.40) that $f$ decays as in (3.9) as $r \rightarrow \infty$, this (fast) decay being that required to specify a legitimate second-kind solution.

\subsection{Summary: $n>0$}

The Cauchy problem for (3.1) exhibits the following regimes.

(I) $m>1-n$. Finite speed of propagation of the interface occurs, with the large-time behaviour being given by the Barenblatt solution.

(IIa) $N<n+1,1-n-(1+n) / N<m<1-n$. For $-n<m<1-n$ the Cauchy problem is uniquely specified and the large-time behaviour is given by the Barenblatt solution. For $1-n-(1+n) / N<m<-n$, non-uniqueness arises, with non-maximal solutions losing mass to infinity via (3.8) and with the maximal (mass-preserving) solution tending to the Barenblatt solution as $t \rightarrow \infty$.

(IIb) $N>n+1,-n<m<1-n$. For $1-n-(1+n) / N<m<1-n$ solutions to the Cauchy problem conserve mass and their large-time behaviour is given by the Barenblatt solution. For $-n<m<1-n-(1+n) / N$ solutions lose mass to infinity and extinguish in finite time via a similarity solution of the second kind, which is expanding if $m_{s}<m<1-n-(1+n) / N$ and contracting for $-n<m<m_{s}, m_{s}$ being defined in (3.33).

For (VIa), $N<n+1, m<1-n-(1+n) / N$, and (VIb), $N>n+1, m<-n$, instantaneous extinction occurs.

For (3.3) the smoothest compactly supported solutions for $m>1-n$ are as in (I) above. For $m<1-n$ (and we stress that there is here no lower bound on $m$, in contrast to the above results for (3.1)) the corresponding solutions have fixed front with

$$
\begin{aligned}
& p \propto z^{\frac{n+1}{n}} \text { as } z \rightarrow 0^{+} \text {for }-2 n<m<1-n, \\
& p \propto z^{\frac{m+n-1}{m+n}} \text { as } z \rightarrow 0^{+} \text {for } m<-2 n ;
\end{aligned}
$$

given that

$$
p \propto z \text { as } z \rightarrow 0^{+} \text {for } m>1-n,
$$

continuity of exponents in the local behaviour arises as $\gamma \rightarrow 0$ in (3.4), corresponding to $|m| \rightarrow \infty$.

For the Cauchy-Dirichlet problem, infinite-time extinction (3.26) occurs for $m>1-n$, finite-time extinction of the form (3.28) for $-n<m<1-n, N<n+1$ and for $m_{s}<m<$ $1-n, N>n+1$, and finite-time extinction in the form of a (contracting) second-kind similarity solution for $-n<m<m_{s}, N>n+1$. For $m<-n$ instantaneous extinction occurs. 
Each of the above results constitutes a rather direct generalisation of those for $n=1$ (i.e. for PME). As we shall see in the next section, the situation is significantly different when $n<0$.

\section{$4 n<0$}

\subsection{Preliminaries}

Here we concern ourselves with the one-dimensional case

$$
\frac{\partial u}{\partial t}=-\frac{\partial}{\partial x}\left(u^{m}\left|\frac{\partial u}{\partial x}\right|^{n-1} \frac{\partial u}{\partial x}\right)
$$

only, for reasons noted in Section 1 (through the radially symmetric case certainly represents a worthwhile generalisation). A feature of immediate significance associated with the change in sign from (3.1) is that the flux is the direction of increasing $x$ is now

$$
+u^{m}\left|\frac{\partial u}{\partial x}\right|^{n-1} \frac{\partial u}{\partial x}
$$

i.e. up the concentration gradient: this has obvious implications in what follows.

Applying (3.2) gives

$$
\frac{\partial p}{\partial t}=-p \frac{\partial}{\partial x}\left(\left|\frac{\partial p}{\partial x}\right|^{n-1} \frac{\partial p}{\partial x}\right)-\frac{n}{m+n-1}\left|\frac{\partial p}{\partial x}\right|^{n+1} ;
$$

now (3.2) maps zeros of $u$ into those of $p$ for $m<1-n$.

We first revisit the separable form since it appears in a number of guises in what follows. The expression

$$
p^{n}=\left|\frac{n}{n+1}\right|^{n-1} \frac{(m+n-1) x^{n+1}}{(n+1)(m+2 n) t}
$$

provides a legitimate solution in three regimes:

(III) $-1<n<0, m<-2 n$. Here $p$ and $u$ are both unbounded at $x=0$ and zero at infinity.

(V) $n<-1,1-n<m<-2 n$. In this case $p$ is zero and $u$ unbounded at $x=0$ and conversely at infinity.

(VIII) $-1<n<0, m>1-n$. Now $p$ is unbounded and $u$ zero at $x=0$ and conversely at infinity.

We again note the exceptional status of $(m, n)=(2,-1)$ within the above. In the remaining ranges of $(m, n)$ the solution

$$
p^{n}=\left|\frac{n}{n+1}\right|^{n-1} \frac{(1-m-n) x^{n+1}}{(n+1)(m+2 n)\left(t_{c}-t\right)} \quad t<t_{c},
$$

is viable and may represent finite-time extinction (at $t=t_{c}$ ) or waiting-time phenomena, for example.

The quasi-steady balance will also again prove significant, and involves the following cases 
$(\alpha) \quad-n<m<-2 n$.

$$
u^{m+n} \sim\left(\frac{m+n}{-n}\right)^{n}(-J(t)) x^{n} \text { as } x \rightarrow+\infty
$$

representing an underspecified solution branch with $J<0$ (flux in from infinity)

( $\beta) \quad m<-n$.

$$
u^{m+n} \sim\left(\frac{m+n}{n}\right)^{n}(-J(t)) z^{n} \text { as } z \rightarrow 0^{+}
$$

gives a Stefan-type solution branch as, though $J<0$ is again required, i.e. mass is gained rather than lost at $x=s(t)$.

$$
\begin{aligned}
(\gamma) m & >-2 n \\
u^{m+n} & \sim\left(\frac{m+n}{-n}\right)(-J(t))\left|x-X_{1}\right|^{n} \text { as } x \rightarrow X_{1},
\end{aligned}
$$

with $J<0$ (i.e. flux into $x=X_{1}$ ), represents a possible correctly specified singular behaviour as $x \rightarrow X_{1} ; X_{1}$ will denote the location of a maximum in the initial data and $J$ will in general take different values as $x \rightarrow X_{1}^{ \pm}$.

Next we consider the local behaviour at a maximum or minimum of $u$, located at $x=\sigma(t)$ with $u_{\sigma}(t) \equiv u(\sigma(t), t)$. Setting

$$
u \sim u_{\sigma}(t)+U(z, t), x=\sigma(t)+z
$$

implies (we briefly revert to (2.1) here since we wish also to note the implications for $n>0$ )

$$
\dot{u}_{\sigma}-\dot{\sigma} \frac{\partial U}{\partial z} \sim \frac{1}{n} u_{\sigma}^{m} \frac{\partial}{\partial z}\left(\left|\frac{\partial U}{\partial z}\right|^{n-1} \frac{\partial U}{\partial z}\right) .
$$

Now

$$
U \sim A(t)|z|^{\frac{n+1}{n}} \quad \text { as } \quad z \rightarrow 0^{+}
$$

with $A$ given by

$$
u_{\sigma}^{-m} \dot{u}_{\sigma}=\left|\frac{n+1}{n}\right|^{n-1} \frac{n+1}{n^{2}}|A|^{n-1} A
$$

is a legitimate local balance for $n>0$ (whereby $u_{\sigma}$ is increasing at a minimum of $u, A>0$, and decreasing at a maximum, $A<0 ;(4.11)$ contains the requisite two degrees of freedom (namely $\sigma$ and $\left.u_{\sigma}\right)$ ) but for $n<-1$, where the expansion (4.8) might still appear to be self-consistent given (4.10), the $\dot{\sigma}$ term in (4.9) would dominate and the only viable scenario for $n<-1$ would seem to have $\dot{\sigma}=0$, so that $\sigma(t) \equiv X_{1}$, and

$$
\begin{aligned}
& u \sim u_{\sigma}^{+}(t)+A^{+}(t) z^{\frac{n+1}{n}} \quad \text { as } \quad z \rightarrow 0^{+}, \\
& u \sim u_{\sigma}^{-}(t)+A^{-}(t)(-z)^{\frac{n+1}{n}} \quad \text { as } \quad z \rightarrow 0^{-},
\end{aligned}
$$


where the pairs $\left(u_{\sigma}^{ \pm}, A^{ \pm}\right)$each satisfy (4.11), restoring the required two degrees of freedom (in the form of $u_{\sigma}^{ \pm}$) and implying that $u$ increases at a maximum and decreases at a minimum, in sharp contrast to the conventional case. Thus for $n<-1$ the maxima and minima of $u$ are fixed at their initial locations, and at these places $u$ develops discontinuities (cf. [16], [4] for $m=0$ and [13] for the corresponding differentiated version in which discontinuities correspond to persisting delta functions; in contrast to these analyses, however, we are concerned here with cases in which $A^{ \pm}$take the same sign), the sides $z \lessgtr 0$ decoupling from one another and with (4.12) amounting to no-flux boundary conditions on each side. A (positive) minimum of $u$ will touch down to form a zero (see below). The corresponding solution branches for $-1<n<0$ exhibit even more extreme behaviour: again, see below (it is noteworthy that behaviour for $n<-1$ is in some respects more benign than that in the apparently less-singular regime $-1<n<0)$.

The above local balances provide the ingredients we need to analyse the Cauchy problem for (4.1), which we do in the next three subsections. Since a mass-preserving interface, if present, will have $\dot{s} \leq 0$, the Cauchy-Dirichlet problem is of limited interest for $n<0$.

\subsection{The Cauchy problem: $n<-1$}

For $m<1-n$ (we note that there is no lower bound on $m$ here) and $1-n<m<-2 n$ the Barenblatt solutions describe possible large-time behaviours (compactly supported in the former case and with an algebraically decaying tail in the latter) for initial data of finite mass, but there is a very important qualification. If the initial data contain a single maximum, and no minima, the argument at the end of the previous subsection implies that the largetime behaviour comprises two separate 'half-Barenblatt' solutions, one either side of the initial maximum, with (in general) a discontinuity there (there is no interaction between the two sides - in the case that the two sides of the maximum contain the same mass in the initial data, this large-time similarity solution will itself be continuous, but the correction terms will in general not be, though symmetric initial data will not of course generate a discontinuity). If the initial data contain a minimum (and hence multiple maxima) then for $m<1-n$ touch down will occur (in general not at the same time on the two sides of the minimum) necessitating further copies of the (contracting) half-profile similarity solutions in describing the $t \rightarrow \infty$ asymptotics, with a dead core $(u \equiv 0)$ between them that is initiated at the location of an initial minimum. For $1-n<m<-2 n$ a minimum at $x=X_{0}$ will instead lead to separable solutions, of the form

$$
u \sim t^{-\frac{1}{m+n-1}} f(x) \quad \text { as } \quad t \rightarrow \infty
$$

occupying each of the domains $X_{1}<x<X_{0}$ and $X_{0}<x<X_{2}$, where $X_{1}$ and $X_{2}$ are the neighbouring maxima in the initial data, with $f(x)$ having singular behaviour local to these maxima, as in (4.3) (with $x$ replaced by $\left|x-X_{i}\right|$ for $i=1,2$ ), thereby matching with the tails of the Barenblatt solutions that contract down onto $x=X_{1}$ and $x=X_{2}$. 
For $m>-2 n$ there seem to be no viable finite-mass large-time solutions, suggesting that the concentration of all the mass into the same number of delta functions as there are maxima in the initial data occurs. Since there is apparently no suitable far-field behaviour, this presumably happens instantaneously if there is no minimum between a maximum and infinity; if there is such a minimum (at $x=X_{0}$ ), however, it seems plausible that the behaviour at a maximum (at $x=X_{1}$ ) will switch from (4.12) to the singular form (4.7) at some finite time (plausibly via a similarity solution of the second kind), thereby generating a delta function at the $x=X_{1}$, and that the large-time behaviour will be of the form (4.13) in $X_{1}<x<X_{0}$, characterising how the mass there all ultimately drains into a delta function at $x=X_{1}$.

\subsection{The Cauchy problem: $-1<n<0$}

For $m<-2 n$, finite-mass (and mass-conserving) singular behaviour of the form (4.3) is available at a maximum of $u$, with no transfer of mass possible between the two sides, and with the support contracting, as in (2.9). We conjecture that the singular Barenblatt solution (2.7) describes the large-time behaviour (multiple half-copies will again be needed, as may dead cores, their number depending on the number of stationary points in the initial data).

For $-2 n<m<1-n$, drainage into the maxima in the form (4.7) is appropriate, with travelling-wave balance (2.9) holding at the contracting interfaces. We conjecture that all the mass drains into one or more delta functions in finite time, the ultimate behaviour being described by similarity solution of the second kind.

For $m>1-n$ the two local behaviours available are again (4.7) (drainage into the maxima) and (4.3), which will, with $x$ replaced by $\left(s_{0}-x\right)$, characterise the solution at a (fixed) interface and which will instantly emerge at any local minima. The large-time behaviour is then expected to comprise separable solutions (4.13) transferring the remnants of the mass into the delta function(s).

\subsection{The Cauchy problem: $n=-1$}

This borderline is worthy of special (but brief) treatment because the linearising transformation recorded in Appendix 1 simplifies the analysis significantly. Thus, inverting the roles of $x$ and $u$ gives

$$
\frac{\partial x}{\partial t}=\frac{\partial}{\partial u}\left(u^{m} \frac{\partial x}{\partial u}\right),
$$

from which the status of $m=2$ as a borderline value will be apparent. For $m<2$ the source solution

$$
x \sim t^{-\frac{1}{2-m}} F(\xi), \xi=u / t^{\frac{1}{2-m}}
$$

pertains at large time (taking the initial data to contain a single maximum at $x=0$ ), implying

$$
F(\xi)=\eta_{0} \exp \left(-\xi^{2-m} /(2-m)^{2}\right)
$$


for some constant $\eta_{0}>0$ (for $x>0$ ) and hence

$$
u \sim t^{\frac{1}{2-m}} f(\eta), \eta=x t^{\frac{1}{2-m}}
$$

with

$$
f^{2-m}(\eta)=(2-m)^{2} \ln \left(\eta_{0} / \eta\right)
$$

The interface location $\eta=\eta_{0}$ is determined by the mass of the initial data in $x>0$ and an equivalent expression holds in $x<0$; $u$ thus has a logarithmic singularity at $x=0$, as might be expected for a borderline case.

For $m>2$ the front is fixed at its initial location (or at the location of a minimum in the initial data) $x=s_{0}$, so the similarity reduction

$$
x \sim F(\xi), \quad \xi=u t^{\frac{1}{m-2}}
$$

is appropriate for large $t$, yielding in $x>0$

$$
F(\xi)=s_{0}\left(1-\Gamma\left(\frac{m-1}{m-2}, \frac{1}{(m-2)^{2} \xi^{m-2}}\right) / \Gamma\left(\frac{m-1}{m-2}\right)\right),
$$

where $\Gamma(\bullet, \bullet)$ denotes the incomplete gamma function and we have again located the maximum at $x=0$. More transparently,

$$
\frac{d F}{d \xi}(\xi)=-\frac{s_{0}}{\Gamma\left(\frac{m-1}{m-2}\right)(m-2)^{\frac{m}{m-2}} \xi^{m}} \exp \left(-1 /(m-2)^{2} \xi^{m-2}\right) .
$$

From this we recover the separable form

$$
u \sim t^{-\frac{1}{m-2}} f(x)
$$

wherein $f$ approaches zero logarithmically at the fixed front,

$$
f^{-(m-2)}(x) \sim(m-2)^{2} \ln \left(1 /\left(s_{0}-x\right)\right) \quad \text { as } \quad x \rightarrow s_{0}^{-},
$$

and

$$
f^{m-1}(x) \sim \frac{s_{0}}{\Gamma\left(\frac{m-1}{m-2}\right)(m-1)(m-2)^{\frac{m}{m-2}} x} \quad \text { as } \quad x \rightarrow 0^{+}
$$

governing the drainage of mass into a delta function at the origin.

Finally, in the highly exceptional case $(m, n)=(2,-1)$ we have (through mapping (4.14) to the heat equation)

$$
x \sim \frac{1}{2} s_{0} \operatorname{erfc}\left((\ln u+t) / 2 t^{\frac{1}{2}}\right) \quad \text { as } \quad t \rightarrow \infty
$$

setting $c=\ln u+t$ in (4.1) yields

$$
\frac{\partial c}{\partial t}=-\frac{\partial}{\partial x}\left(\left|\frac{\partial c}{\partial x}\right|^{-2} \frac{\partial c}{\partial x}\right)
$$


(the same transformation is applicable to higher dimensions also) so, in terms of $c$, (4.15) corresponds to the similarity reduction

$$
c=t^{\frac{1}{2}} h(x)
$$

but in terms of $u$ it appears more exotic. From (4.15) we obtain the local behaviours

$$
\begin{aligned}
& u \sim \exp \left(-t+2(t \ln (1 / x))^{\frac{1}{2}}\right) \quad \text { as } \quad x \rightarrow 0^{+}, \\
& u \sim \exp \left(-t-2\left(t \ln \left(1 /\left(s_{0}-x\right)\right)\right)^{\frac{1}{2}}\right) \quad \text { as } \quad x \rightarrow s_{0}^{-},
\end{aligned}
$$

i.e. $u$ is unbounded as $x \rightarrow 0^{+}$and tends to zero as $x \rightarrow s_{0}^{-}$; the complexity of those expressions relates to the borderline status of the exponents. The solution (4.15) conserves mass, which is concentrated as $t \rightarrow \infty$ over the exponentially narrow range $\eta=O(1)$, whereby

$$
u \sim t^{-\frac{1}{4}} e^{t} f(\eta), \quad x=t^{\frac{1}{4}} e^{-t} \eta \quad \text { as } \quad t \rightarrow \infty \quad \text { with } \quad \eta=O(1)
$$

and

$$
f(\eta)=1 / 2 a \pi^{\frac{1}{2}} \eta
$$

This behaviour is indicative of the transition to a delta function at a maximum noted in the parameter regimes above and would be significantly harder to derive without appeal to the linearising transformation.

\subsection{Compactly supported solutions to (4.2)}

Since (3.2) maps zeros of $u$ into those of $p$ when $m<1-n$, the results of the previous subsections then continue to apply here to the smoothest solution branches and those of Section 4.6 to the underspecified ones. For $m>1-n$, however, we become interested in solutions for which $u$ is unbounded at the interface. In this regime correctly specified solution branches have fixed fronts and the following subcases arise, occupying the remainder of $(m, n)$ parameter space.

(V) $\quad-1-n<m<-2 n \quad$ (so that $n<-1$ ).

The local form (4.3) applies at a fixed front and (4.12) at the maximum; a subdivision occurs if the initial data contain a local minimum, this also applying in the cases below.

(VIII) $n>-1, m>1-n$.

(4.7) again holds at a fixed front, while (4.3), which is singular in terms of $p$, holds at the initial maximum.

(IX) $n<-1, m>-2 n$.

(4.7) (which has $p \rightarrow 0$ ) holds at a fixed front and (4.12) at the maximum.

The underspecified solution branches for $m>1-n$ have $\dot{s}>0$ (contrast the discussion in Section 3.3), with (compare (3.17))

$$
p \sim((m+n-1) \dot{s} /(-n))^{\frac{1}{n}} \text { as } z \rightarrow 0^{+} .
$$

Interestingly, these are smoother than the correctly specified branches just outlined, for which $\partial p / \partial x$ is unbounded at the interface. 


\subsection{Stefan-type solution branches}

In terms of (4.1), the underspecified solution branch (4.6) is available for $m<-n$, in which case the mass-preserving branch is the smoothest one. It is noteworthy that $J<0$ is required here, so any Stefan-like boundary condition prescribed in formulating a correctly specified problem must involve mass gain, rather than mass loss; the properties of the resulting solutions will not be explored here. We remark that solutions with $\dot{s}>0$ can occur (driven by gain of mass at the interface), as they can on the underspecified solution branch in Section 4.5, whereas the correctly specified branches have $\dot{s} \leq 0$. Via (4.5), this branch can be continued into $-n<m<-2 n$ as a class of solutions that gain mass from infinity (analogous to the non-maximal solutions in $u>0$ referred to in Section 3, and which we shall accordingly term 'non-minimal'); in this range the Cauchy problem for (4.1) is thus correctly specified on imposition of a zero-flux condition at infinity (rather than this holding automatically, as it does for smaller $m$ ). There are further qualitative differences from the non-maximal case of Section 3: here the non-minimal solutions can coexist with compactly supported ones (leading to non-compactly supported solutions to (4.2); none such arise in the analysis of (3.3)), namely in $-n<m<1-n$, and there is a range beyond that of the non-minimal solutions, namely $-2 n<m<1-n$ (so that $n>-1$ ), in which uniqueness for the Cauchy problem is reinstated - in this latter regime it is appropriate to think of (4.4) as usurping the quasi-steady balance that is no longer applicable: (4.4) can be viewed as describing finite-time compactification from initial data having a suitable algebraically decaying tail (see also (2.14)).

\subsection{Summary: $n<0$}

We shall give particular attention here to cases which have no analogue for PME or PLE. Our conjectures are as follows.

For the Cauchy problem for (4.1) the following cases arise. For (IV), $n<-1, m<1-n$, finite speed of propagation occurs as in (2.11), two half-copies of the (contracting) Barenblatt solution (2.5) describing the large-time behaviour, one either side of the maximum in the initial data and with no transfer of mass between them; if the initial data contain more than one maximum then additional half copies are needed with dead cores ultimately present between the maxima. This regime encompasses PLE $(m=0)$ for $n<-1$. For $(\mathrm{V}), n<-1,1-n<$ $m<-2 n$, the Barenblatt solution decays algebraically at infinity (see (4.3)) and a possible large-behaviour comprises two half copies of (2.6), one either side of the initial maximum; if there are multiple maxima, two half-copies of a separable solution (4.13) are additionally present either side of the intervening minimum. Because of the uphill diffusion property of (4.1), such behaviour presumably requires that the initial data decay slower than the above algebraic tail, plausibly with non-existence associated with delta-function formation otherwise arising, and there are particular unresolved issues in this case (a number of questions concerning instantaneous and finite-time blow up would certainly seem of more general relevance in the 
current context). For (IX), $n<-1, m>-2 n$, delta functions form at the maxima in the initial data, instantaneously if minima are not present but after some finite time if they are. For $-1<n<0$ bounded behaviour with $u>0$ does not seem possible at a maximum or minimum (contrast (4.12)) and it seems likely that any initial minimum will instantly touch down; for (III), $-1<n<0, m<-2 n$, constant mass solutions with contracting interfaces are available, with half copies of the Barenblatt soution furnishing the large-time behaviour, while for larger $m$ the mass will drain into delta functions at the initial maxima (with contracting interfaces for (VII), $-1<n<0,-2 n<m<1-n$, and fixed ones for (VIII), $-1<n<0, m>1-n)$. The regime (VII) is perhaps of particular interest in that the intermediate-asymptotic behaviour (loss of all mass at finite time into a delta function) is postulated to be similarity solution of the second kind: such behaviour is not exhibited in the one-dimensional case in any of the other regimes (including those with $n>0$ ).

Turning now to (4.2) with $m>1-n$, so that zeros of $p$ corresponding to $u$ being unbounded, the correctly specified branches all have fixed interfaces and are as follows: for $n<-1$ both for (V), $1-n<m<-2 n$, and for (IX), $m>-2 n$, the solution is bounded, the local behaviour at the interface differing between the two, while for $n>-1$, (VIII), the solution is unbounded at the initial maximum. In all these cases, the large-time behaviour is expected to be of the separable form (4.13).

The regimes in which no finite-mass solutions exist for $t>0$ are hence (VI), $n>0, m<-2 n$, (instantaneous loss to infinity) and (IX), $n<-1, m>-2 n$, when the initial data contain a single maximum (instantaneous loss into a delta function located at the maximum).

\section{Discussion}

Part of the motivation for the current work comes from that of [2] and [3], which focus on the case, in the current notation, of $m=0, n<0$ (especially $n<-1$ ), with the image intensity $u$ satisfying $0 \leq u \leq 1$ and with, in particular, $u \rightarrow 0$ as $x \rightarrow-\infty$ and $u \rightarrow 1$ as $x \rightarrow+\infty$, corresponding to the edge of an image, the property of edge enhancement being a key feature of the case $n<-1$. We have chosen here instead to focus in effect on localised regions of image intensity, but note, firstly, that the classification of the local behaviour at the edge of the support of $u$ is independent of the form of the initial data and, secondly, that a generalisation of (1.1) (in one dimension) to

$$
\frac{\partial u}{\partial t}=\frac{1}{n} \frac{\partial}{\partial x}\left(u^{m_{1}}(1-u)^{m_{2}}\left|\frac{\partial u}{\partial x}\right|^{n-1} \frac{\partial u}{\partial x}\right)
$$

might be instructive in the edge context (of course necessitating that $u$ be normalised in such a way that $u_{0} \in[0,1]$ is indeed ensured); a starting point on (5.1) would be an investigation of the similarity reduction

$$
u=f(\xi), \xi=x / t^{\frac{1}{n+1}}
$$


from which it is clear that $n<-1$ is required by edge sharpening.

A key outcome of the current work is that, while the behaviour of (3.1) (i.e. for $n>0$ ) with $m \neq 1$ involves rather natural generalisations of PME and PLE properties, that of (4.1) (i.e. for $n<0)$ can differ significantly. Indeed, the regimes (V)-(IX) of Section 2 do not intersect the $m=0$ axis and the behaviour within them cannot therefore be extrapolated from that of PLE. Amongst the most striking of these features, we note the following. For (4.1), regime (VII) is noteworthy in that the intensity is conjectured to completely localise (as a delta function) in finite time, whereas for (VIII) this seems to occur only as $t \rightarrow \infty$, while for (III) solutions conserve mass, albeit being singular at the initial maxima. Since regime (VII) lies between (III) and (VIII), this postulated finite-time behaviour is somewhat counterintuitive. The implications, if any, of such properties in applications to image analysis have yet to be explored. Moreover, we have little doubt that the issues studied need considerable further mathematical attention.

We conclude with a number of naive observations. The regime $n<0$ is noteworthy in the image-analysis context in the sense that regions of high intensity will be further strengthened and the profile will sharpen (with $\dot{s} \leq 0$ ), properties in sharp contrast to those of conventional diffusion. Moreover, we emphasise that even the qualitative behaviour depends significantly on the value of $m$ and, for $m>1-n$, on whether the formulation (4.1) or (4.2) is adopted (with $u=0$ and $p=0$, respectively, at the moving boundary): properties not encompassed by the analyses of [2], [3] are accessible thereby. That the Barenblatt solutions for $n<0$ contract to a delta function as $t \rightarrow \infty$, rather than starting from one, presumably affects the application of rescaling arguments in showing convergence; much of the linear-stability analysis would seem to proceed in the usual fashion, however. We have sought to emphasise the efficacy of piecing together simple local analyses in characterising solutions; the borderline cases we have identified in this way are (i) $n=0$, (ii) $n+1=0$, (iii) $m+n=0$, (iv) $m+n-1=0$ and (v) $n+1+N(m+n-1)=0$. Cases where these coincide are of particular significance - for example, (iii) and (v) coincide at $m=N-1, n=-(N-1)$, which played a notable role in Section 3.2. There are two cases with $N>0$ in which three are coincident, namely (ii), (iv) and (v) at $m=2, n=-1$ for all $N$ (cf. Section 4.4) and (i), (iii) and (v) at $m=0, n=0, N=1$ (see Appendix 2): these cases are entirely exceptional.

\section{Acknowledgements}

This work was completed while the author was a Visiting Fellow at the Isaac Newton Institute, Cambridge: the funding of the Simons Foundation is gratefully acknowledged. 


\section{Appendix 1 Equivalence transformations}

\section{A1.1 The one-dimensional case}

The results of this subsection amount to a special case of Table 2 of [11]. Starting from

$$
\frac{\partial u}{\partial t}=\operatorname{sgn}(n) \frac{\partial}{\partial x}\left(u^{m}\left|\frac{\partial u}{\partial x}\right|^{n-1} \frac{\partial u}{\partial x}\right)
$$

and introducing $v$ and $y$ through

$$
u=\frac{\partial v}{\partial x}, \quad y=\frac{\partial x}{\partial v}=1 / u
$$

gives (without loss of generality)

$$
\frac{\partial v}{\partial t}=\operatorname{sgn}(n)\left(\frac{\partial v}{\partial x}\right)^{m}\left|\frac{\partial^{2} v}{\partial x^{2}}\right|^{n-1} \frac{\partial^{2} v}{\partial x^{2}}
$$

a hodograph transformation then yields

$$
\frac{\partial x}{\partial t}=\operatorname{sgn}(n)\left(\frac{\partial x}{\partial v}\right)^{1-3 n-m}\left|\frac{\partial^{2} x}{\partial v^{2}}\right|^{n-1} \frac{\partial^{2} x}{\partial v^{2}}
$$

as the governing equation for $x(v, t)$, so that

$$
\frac{\partial y}{\partial t}=\operatorname{sgn}(n) \frac{\partial}{\partial v}\left(y^{1-3 n-m}\left|\frac{\partial y}{\partial v}\right|^{n-1} \frac{\partial y}{\partial v}\right)
$$

i.e. the pairs of exponents $(m, n)$ and $(1-3 n-m, n)$ map into each other (for $m=(3 n+1) / 2$ the equation maps into itself).

Instead applying a hodograph transformation directly to (A1.1) reveals that $x(u, t)$ satisfies

$$
\frac{\partial x}{\partial t}=-\operatorname{sgn}(n) \frac{\partial}{\partial u}\left(u^{m}\left|\frac{\partial x}{\partial u}\right|^{-n-1} \frac{\partial x}{\partial u}\right)
$$

the case $n=1$ thus maps to a linear diffusion equation (see also (A1.4) and (A1.5) below). Now introducing

$$
\rho=\frac{n-1}{n+m-1} u^{\frac{n+m-1}{n-1}}
$$

for $n \neq 1, n+m \neq 1$, yields

$$
\frac{\partial x}{\partial t}=-\operatorname{sgn}(n) \frac{1}{\rho^{D-1}}\left(\rho^{D-1}\left|\frac{\partial x}{\partial \rho}\right|^{-n-1} \frac{\partial x}{\partial \rho}\right), \quad D=\frac{n-1}{n+m-1},
$$

i.e. the radially symmetric PLE in $D$ dimensions.

Introducing

$$
\xi=\frac{\partial x}{\partial u}
$$


leads from (A1.3) to

$$
\frac{\partial \xi}{\partial t}=-\operatorname{sgn}(n) \frac{\partial^{2}}{\partial u^{2}}\left(u^{m}|\xi|^{-n-1} \xi\right)
$$

and hence for $n \neq 0, m \neq-2 n$ to the radially symmetric $\mathrm{PME}^{2}$

$$
\frac{\partial \zeta}{\partial t}=-\operatorname{sgn}(n) \frac{1}{r^{N-1}} \frac{\partial}{\partial r}\left(r^{N-1} \frac{\partial}{\partial r}\left(|\zeta|^{-n-1} \zeta\right)\right), N=\frac{2(n+m)}{2 n+m},
$$

where

$$
\xi=u^{\frac{m}{n}} \zeta, r=\frac{2 n}{2 n+m} u^{\frac{2 n+m}{2 n}}
$$

Applying the corresponding transformations to (A1.2) instead, we obtain dimensions

$$
D^{\prime}=-\frac{(n-1) D}{(n+1) D+n-1}, N^{\prime}=\frac{2(N-2+2 n)}{(n+1) N-2},
$$

the latter corresponding to local transformations from [12].

A special case arises when $3 n+m=1$, as in Table 1 of [11]. In this case (A1.2) becomes the one-dimensional PLE and setting

$$
z=\frac{\partial y}{\partial v}
$$

gives

$$
\frac{\partial z}{\partial t}=\operatorname{sgn}(n) \frac{\partial^{2}}{\partial v^{2}}\left(|z|^{n-1} z\right)
$$

being of the class (A1.1) with $n=1$, thus allowing the various transformations above to be repeated starting from the differentiated form (A1.7). Under the potential-hodograph transformation (A1.7) maps into another equation of the same class, and in terms of the exponents $(m, n)$ the following six special cases can be mapped into each other (cf. Table 1 of [11]): $(1-3 n, n),(0, n),(n-1,1),(-n-1,1),(0,-n),(1+3 n,-n)$.

\section{A1.2 The radially symmetric case}

Unsurprisingly, the possibilities are more limited for the $N$-dimensional radial equation

$$
\frac{\partial u}{\partial t}=\operatorname{sgn}(n) \frac{1}{r^{N-1}} \frac{\partial}{\partial r}\left(r^{N-1} u^{m}\left|\frac{\partial u}{\partial r}\right|^{n-1} \frac{\partial u}{\partial r}\right) .
$$

Setting

$$
x=r^{N}
$$

\footnotetext{
${ }^{2}$ We stress that the dimension $N$ differs from $D$ in (A1.4), except when $N=1$ or when $n=-(N-2) /(N+2)$, the latter corresponding to $N=N^{\prime}, D=D^{\prime}$ in the transformations noted below. Indeed, $D=-(n-1)(N-$ $2) /((n+1) N-2), N=2(D+n-1) /((n+1) D+n-1)$; in the linear case, $n=-1$, these become $N+D=2$.
} 
gives, up to a scaling factor

$$
\frac{\partial u}{\partial t}=\operatorname{sgn}(n) \frac{\partial}{\partial x}\left(x^{(n+1)(N-1) / N} u^{m}\left|\frac{\partial u}{\partial x}\right|^{n-1} \frac{\partial u}{\partial x}\right)
$$

so that

$$
\frac{\partial v}{\partial t}=\operatorname{sgn}(n) x^{(n+1)(N-1) / N}\left(\frac{\partial v}{\partial x}\right)^{m}\left|\frac{\partial^{2} v}{\partial x^{2}}\right|^{n-1} \frac{\partial^{2} v}{\partial x^{2}}
$$

and $x(v, t)$ satisfies the triply nonlinear 'one-dimensional' equation

$$
\frac{\partial x}{\partial t}=\operatorname{sgn}(n) x^{(n+1)(N-1) / N}\left(\frac{\partial x}{\partial v}\right)^{1-3 n-m}\left|\frac{\partial^{2} x}{\partial v^{2}}\right|^{n-1} \frac{\partial^{2} x}{\partial v^{2}}
$$

$3 n+m=1$ is again a special case. The significance of (A1.10) in the current context is not apparent, though it does highlight

$$
\frac{\partial w}{\partial t}=w^{m}|\triangle w|^{n-1} \triangle w
$$

as a model doubly nonlinear equation distinct from that addressed herein.

We note from (A1.9) that for $n=-1$ all dimensions $N$ can be mapped into the onedimensional problem and hence into one another. In contrast to (A1.10), $x(u, t)$ does satisfy an equation of the same class as (A1.9), namely

$$
\frac{\partial x}{\partial t}=-\operatorname{sgn}(n) \frac{\partial}{\partial u}\left(u^{m} x^{(n+1)(N-1) / N}\left|\frac{\partial x}{\partial u}\right|^{-n-1} \frac{\partial x}{\partial u}\right),
$$

so (as it reducibility to the one-dimensional problem already implies) the special case $n=-1$ transforms to a linear PDE. This transformation between (A1.9) and (A1.11) shows that (A1.8) can be mapped into another equation of the same class with, in particular, $n$ mapping to $-n$.

\section{Appendix $2 \quad n=0$}

This special case is of very apparent interest, both because the order reduces in the onedimensional case on which we focus (as it does for radial symmetry) and because of its borderline status between the regimes of Sections 3 and 4, implying in particular that both signs are of interest (a comment that is again specific to the one-dimensional case). We for brevity take initial data $u_{0}(x)$ symmetric in $x$, with $u_{0}=0$ for $x \geq s_{0}, d u_{0} / d x \leq 0$ in $x>0$, describe the behaviour in $x>0$ only and start with the choice

$$
\frac{\partial u}{\partial t}=\frac{\partial}{\partial x}\left(u^{m}\left|\frac{\partial u}{\partial x}\right|^{-1} \frac{\partial u}{\partial x}\right)
$$

of sign, leading to a complementary problem in $x>0$ :

$$
\frac{\partial u}{\partial x}=0 \quad \text { or } \quad \frac{\partial u}{\partial t}+m u^{m-1} \frac{\partial u}{\partial x}=0 ;
$$


both of these are of first order - the second-order nature of the original problem is reflected in there being two. For $m>1$ shock formation will occur with (conserving mass at the shock)

$$
u \sim a t^{-1 / m}, \quad s(t) \sim m a^{m-1} t^{1 / m} \text { as } t \rightarrow \infty
$$

for some constant $a$, the exponents being consistent with the Barenblast solution. For $m=1$ we have $s(t)=s_{0}+t$ and, introducing $S(t)<s(t)$, in $x>0$ we have

$$
u=U(t) \text { for } \quad 0<x<S(t), \quad u=u_{0}(x-t) \text { for } S(t)<x<s(t)
$$

where $U(t)$ and $S(t)$ are given by

$$
U=u_{0}(S-t), \quad U S+\int_{S-t}^{s_{0}} u_{0}(x) d x=\text { constant }
$$

so that

$$
U \sim a t^{-1}, \quad S \sim t \quad \text { as } t \rightarrow \infty
$$

For $0<m<1$ the large-time behaviour follows most directly from the similarity reduction

$$
u=t^{-1 / m} f(\eta), \quad \eta=x / t^{1 / m}
$$

whereby

$$
f=\left(\eta_{0} / m\right)^{\frac{1}{m-1}} \quad 0<\eta<\eta_{0}, \quad f=(\eta / m)^{-\frac{1}{1-m}} \quad \eta>\eta_{0}(c f[])
$$

for some positive constant $\eta_{0}$. The above cases clearly reflect the behaviour for $n>0$. Finally (there being no suitable finite-mass solution for $m<0$, i.e. instantaneous extinction occurs), for $m=0$ (cf. [6])

$$
\frac{\partial u}{\partial x}=0 \quad \text { or } \quad \frac{\partial u}{\partial t}=0
$$

and there is unit flux (mass loss) out through the interface, so that

$$
u=U(t) \quad 0<x<S(t), \quad u=u_{0}(x) \quad S(t)<x<s_{0}
$$

where $U(t)$ and $S(t)$ are given by

$$
U=u_{0}(S), \quad \frac{d}{d t}\left(U S+\int_{S}^{s_{0}} u_{0}(x) d x\right)=-1, \quad S(0)=0
$$

exhibiting extinction in finite time.

We now turn to

$$
\frac{\partial u}{\partial t}=-\frac{\partial}{\partial x}\left(u^{m}\left|\frac{\partial u}{\partial x}\right|^{-1} \frac{\partial u}{\partial x}\right)
$$


for which the behaviour will be reminiscent of that for $n<0$. The first of (A2.1) plays no role in this case, while the sign of the second (and hence the direction in which characteristics propagate) changes (see (A2.4) below). For $m>1, u(x, t)$ is given by

$$
u=u_{0}\left(x+m u^{m-1} t\right)
$$

in $0<x<s_{0}$, with $s(t)=s_{0}$, and a delta function is present at $x=0$ that contains the mass lost by (A2.3). For $m=1$

$$
u=u_{0}(x+t), \quad s(t)=s_{0}-t \quad \text { for } t<s_{0},
$$

all the mass being lost into the delta function at the origin by $t=s_{0}$. For $0<m<1$ shock formation occurs, with (A2.2) holding in $0<x<s(t)$ and with $s(t)$ given (to conserve mass there) by

$$
u^{m-1}(s, t)=-\dot{s}
$$

wherein $s<0$; mass again piles up into a delta function at the origin, all of it doing so in finite time. How the discontinuity at $x=s(t)$ is smoothed for small $-n$ is clear from the local solution (2.9). In each of the above cases

$$
\frac{d}{d t} \int_{0}^{s} u(x, t) d x=-u^{m}(0, t)
$$

holds, thereby determining the rate of growth of the delta function at the origin due to the mass flux in from $x>0$. For $m=0, u=u_{0}(x)$ holds in $x>0$, but there is now unity flux in (mass gain) from the interface: this reappears as a growing delta function at the origin. For $m<0$ a singular Barenblatt solution is expected to govern the large time behaviour, whereby in $(\mathrm{A} 2.2)$

$$
f=(\eta /(-m))^{\frac{1}{1-m}} \quad \eta<a, \quad f=0 \quad \eta>a, \quad s(t) \sim a t^{-1 / m},
$$

again with a discontinuity at the interface (how this is smoothed for small $-n$ is clear from (2.7)). Note that in this case the characteristics of

$$
\frac{\partial u}{\partial t}-m u^{m-1} \frac{\partial u}{\partial x}=0
$$

carry information into $x>0$ from the singularity at $x=0$.

\section{References}

[1] G.I. Barenblatt. On self-similar motions fo compressible fluid in a porous medium. Prik. Mat. Mekh. 16, 679-698, 1952.

[2] G.I. Barenblatt. Self-similar intermediate asymptotics for nonlinear degenerate parabolic free-boundary problems that occur in image processing. PNAS 98, 12878-12881, 2001. 
[3] G.I. Barenblatt and J.L. Vázquez. Nonlinear diffusion and image contour enhancement. Interfaces Free Bound. 6, 3154, 2004.

[4] M. Bertsch and R. Dal Passo. Hyperbolic phenomena in a strongly degenerative parablic equation. Arch. Rat. Mech. Anal. 117, 349-387, 1992.

[5] M. Bertsch, R. Dal Passo and M. Ughi. Nonuniquness of solutions of a degenerate parabolic equation. Ann. Mat. Pura Appl. 161, 57-81, 1992.

[6] M. Bonforte and A. Figalli. Total variation flow and sign fast diffusion in one dimension. J. Diff. Eqns. 252, 4455-4480, 2012.

[7] M. Bowen and J.R. King. Asymptotic behaviour of the thin film equation in bounded domains. Euro. J. Appl. Math. 12, 135-157, 2001.

[8] J.R. Esteban, A. Rodríguez and J.L. Vázquez. A nonlinear heat equation with singular diffusivity. Comm. PDEs 13, 985-1039, 1998.

[9] J.R. Esteban and J.L. Vázquez. On the equation of turbulent filtration in one-dimensional porous media. Nonlinear Analysis - Theory, Methods \& Applications 10, 1303-1325, 1986.

[10] V. A. Galaktionov and J. R. King. Fast diffusion equation with critical Sobolev exponent in a ball. Nonlinearity 15, 173-188, 2002.

[11] J.R. King. Some non-local transformations between nonlinear diffusion equations. J. Phys. A 23, 5441-5464, 1990.

[12] J. R. King. Local transformations between some nonlinear diffusion equations. J. Austral. Math. Soc. B 33, 321-349, 1992.

[13] J. R. King. "Instantaneous source" solutions to a singular nonlinear diffusion equation. J. Eng. Math. 27, 31-72, 1993.

[14] J. R. King. Self-similar behaviour for the equation of fast nonlinear diffusion. Phil. Trans. Roy. Soc. Lond. A 343, 337-375, 1993.

[15] J. R. King. Asymptotic analysis of extinction behaviour in fast nonlinear diffusion. J. Eng. Math. 66, 65-86, 2010.

[16] P. Rosenau, P.S. Hagan, R.L. Northcutt and P.S. Cohen. Delayed diffusion due to flux limitation. Phys. Lett. A 143, 26-30, 1989.

[17] D. Stan and J.L. Vazquez. Asymptotic behavior of the doubly nonlinear diffusion equation $u_{t}=\triangle_{p} u^{m}$ on bounded domains. Nonlinear Analysis: Theory, Methods \& Applications 77, 1-32, 2013. 
[18] J.L. Vázquez. Nonexistence of solutions for nonlinear heat equations of fast diffusion type. J. Math. Pures Appl. 71, 503-526, 1992.

[19] J.L. Vázquez. The Porous Medium Equation: Mathematical Theory. Clarendon Press, Oxford, 2006.

[20] J.L. Vázquez. Smoothing and Decay Estimates for Nonlinear Diffusion Equations Equations of Porous Medium Type. Clarendon Press, Oxford, 2006. 